\title{
Metoprolol Induced Psoriasis: A Case Report
}

\author{
Syed Zia Inamdar*,1, Pradeepthi Katragadda', Ammu Sasikumar², Akhila Mohan², Raghavendra \\ Vinayakarao Kulkarni ${ }^{1}$
}

1Department of Pharmacy Practice, BLDEA's SSM College of Pharmacy and Research Centre, Vijaypur- 586103 Karnataka, INDIA. 2Pharm D Intern, BLDEA's SSM College of Pharmacy and Research Centre, Vijaypur -586103 Karnataka, INDIA.

\begin{abstract}
Metoprolol, an antihypertensive drug belonging to the class $\beta 1$ receptor blocker inhibits adrenergic receptors in heart muscle cells and decreases heart rate, contractility and cardiac output thereby decreases the blood pressure. Though, the drug have been approved for the treatment of blood pressure and arrhythmias, the safety profile of it is not yet established in a broader sense. A patient with known hypertensive diagnosis and presenting complaints of bilateral swelling over lower limbs since 15 days and skin lesions over the body since 5 days was admitted to the medicine ward of a tertiary care hospital. After careful evaluation of the patient, the skin lesion was found to be chronic plaque psoriasis of a drug induced nature, which was then treated symptomatically. The suspected drug metoprolol was discontinued. A Naranjo causality assessment of the observed reaction reveals a "probable" causal relationship and rated under "moderate" severe adverse reaction category as per Hartwig's severity assessment.
\end{abstract}

Key words: Adverse Drug Reaction, Skin Lesions, Metoprolol, Chronic Plaque Psoriasis.

\section{INTRODUCTION}

$\beta$ blockers are the agents which are used mainly in the treatment of the cardiovascular diseases to decrease morbidity and mortality. ${ }^{1}$ Metoprolol is a cardio-selective $\beta$ 1adrenoceptor blocker used in the management of hypertension, angina and cardiac arrhythmias. The anti-arrhythmic effect of $\beta$-blockers involves decreasing heart rate, minimizing the spontaneous firing of ectopic pacemakers, delaying the conduction and raising the refractory period of the atrioventricular node. There are various cutaneous side effects associated with its use, ranging from pruritus and photosensitivity rash to inducing or exacerbating existing psoriasis. ${ }^{2}$ Psoriasis is a chronic inflammatory skin disorder clinically characterized by erythematous, sharply demarcated papules and rounded plaques covered by silvery micaceous scale. Drugs have several ways in which they can affect the diathesis of psoriasis including precipitation of psoriasis in predisposed and no predisposed individual; exacerbation of pre-existing psoriatic lesions; induction of lesions in clinically normal skin in patients with psoriasis; and growth of treatment-resistant psoriasis. The clinical presentation of druginduced psoriasis spans the spectrum of generalized plaque psoriasis, palmoplantar pustulosiss and erythroderma. The inclusion of nails and scalp makes the distinction of drug-associated psoriasis a clinically difficult diagnosis. Furthermore, the mechanism of action can also involve both immunological and non-immunological pathways. ${ }^{3}$ The most common type of psoriasis is chronic plaque psoriasis comprising about $70 \%$ to $80 \%$ of psoriatic patients. ${ }^{4}$ The drugs that are most strongly linked to plaque psoriasis exacerbation are $\beta$-blockers, also known as $\beta$-adrenergic receptor antagonists. The lag time for the onset of psoriasis development can take up to 12 months. Oral $\beta$-blocker use has been anecdotally involved in erythrodermic psoriasis and palmoplantar pustular psoriasis. ${ }^{5}$ Psoriasis is a disease characterized by T-cell-mediated hyper proliferation of keratinocytes and inflammatory processes and is classified as a TH1 disease. Hypertension is also characterized by increased oxidative stress
DOI: 10.5530/ijopp.12.1.12

Address for correspondence: Dr. S.Z Inamdar, Professor and Clinical Pharmacist Department of Pharmacy Practice, BLDEA's SSM College of Pharmacy and Research Centre, Vijaypur 586103 Karnataka, INDIA.

Phone no: +91-9986666016 Email Id: syedzia.inamdar@ gmail.com

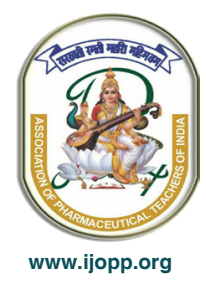


and inflammation and immune mechanisms are reported to be involved in the development of hypertension, with different helper T cells (eg, TH1 and TH2 lymphocytes and T-regulatory cells) participating as pro-and antiinflammatory cells. Chronic inflammation is associated with an increased risk of hypertension according to population based studies. Hence, hypertension maybe associated with psoriasis development because of the shared inflammatory pathways. ${ }^{6}$

\section{CASE REPORT}

A 45 yrs old male patient was presented with the symptoms of bilateral swelling over lower limbs since 15 days and skin lesions over the body [upper and lower limb] since 5 days. Detailed past history suggested that the patient consulted the physician for hypertension for which he was prescribed metoprolol $-100 \mathrm{mg}$ once daily. He also complained about acute skin lesions after 1 year of treatment but it was managed with liquid paraffin and the symptoms subsided after a few days. After nearly 6 years of continuing the medication, the patient now complained of multiple red colored raised skin lesions initially over scalp which was insidious in onset and gradually progressive to involve the trunk and extremities and are associated with itching of moderate grade, the laboratory investigation revealed a marginal elevation of ESR (75), polymorphs (80) and slight decrease in $\mathrm{MCH}$ (28) and lymphocytes (16), Blood pressure $160 / 90 \mathrm{mmHg}$, RBS $177 \mathrm{mg} / \mathrm{dl}$ and GRBS $308 \mathrm{mg} / \mathrm{dl}$.

Multiple therapies were prescribed to the patient. Tab. Cefixime $200 \mathrm{mg}$ was given orally to the patient for prophylaxis 7 days, patient was a known case of diabetes mellitus since $2 \mathrm{yrs}$ and he was on tablet metformin 500mg twice a day. The laboratory values suggested an increase in GRBS levels which made the physician to switch to insulin (6-6-4 IU) therapy on $3^{\text {rd }}$ day of the treatment. Cyprohepatidine- (antihistamine) $4 \mathrm{mg}$ was given orally twice a day for six days as patient was suffering from allergic symptoms; Tab. Metoprolol 100mg was given orally once a day for hypertension for 2 days during hospital stay, but the symptoms continued to worsen. On the $3^{\text {rd }}$ day the patient was cross referred for dermatologist evaluation and opinion, which suggested replacement of metroprolol with telmisartan $40 \mathrm{mg}$ and prescribed $1 \%$ of hydrocortisone and liquid paraffin for topical application to relieve psoriasis inflammation and pruritus. [Figure 1 and Figure 2.]

Indian Journal of Pharmacy Practice, Vol 12, Issue 1, Jan-Mar, 2019

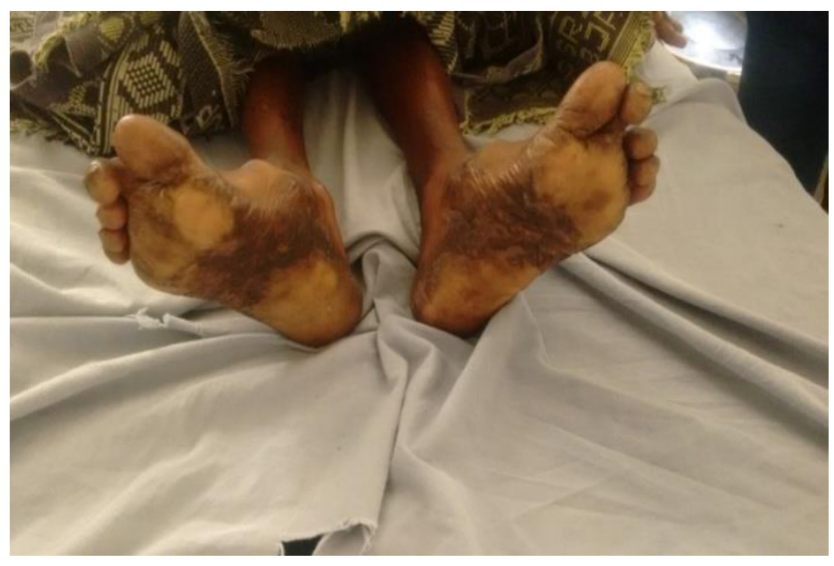

Figure 1: Psoriasiform Drug Eruption: Lower limb.

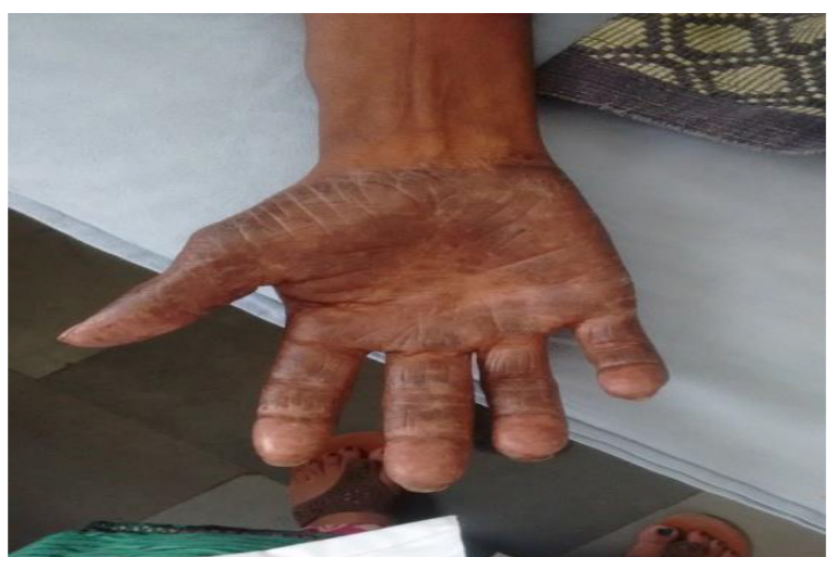

Figure 2: Psoriasiform Drug Eruption: Upper limb.

\section{DISSCUSSION}

Psoriasis is a chronic, immune-mediated inflammatory skin disease that is associated with a high physical and psychosocial burden of disease. Psoriasis governs to have multifaceted etiology. A complex interplay between genetic predisposing factors and environmental factors leads to the onset of psoriasis, infection, physical trauma to the skin (ie, the Koebner phenomenon), emotional stress and exposure to drugs are various factors elicit to an exacerbation of psoriasis. In the current case, the patient was admitted with the complaints of bilateral of both legs since 15 days and skin lesions since 5 days. While assessing the medical/medication history, it was found that the patient was a known case of hypertension and was taking antihypertensive medication metoprolol $100 \mathrm{mg}$ daily as per the advice of physician for treating hypertension for almost 6 years. History also reveals the development of skin lesion after one year of treatment with metoprolol, which was treated then symptomatically. The patient was presented with similar symptoms of skin lesions on upper and lower limb after a gap of 5 years. The skin lesion continued to worsen and a careful dermatological 
Table 1: Causality Assessment of suspected ADR using Naranjo Scale.

\begin{tabular}{|c|c|c|c|c|}
\hline Questions & Yes & No & $\begin{array}{l}\text { Don't } \\
\text { Know/NA }\end{array}$ & Score $^{*}$ \\
\hline Are there previous conclusive reports on this reaction? & +1 & 0 & 0 & +1 \\
\hline Did the adverse event appear after the suspected drug was administered? & +2 & -1 & 0 & +2 \\
\hline $\begin{array}{l}\text { Did the adverse reaction improve when the drug was discontinued or a specific } \\
\qquad \text { antagonist was administered? }\end{array}$ & +1 & 0 & 0 & +1 \\
\hline Did the adverse event reappear when the drug was re-administered? & +2 & -1 & 0 & 0 \\
\hline $\begin{array}{l}\text { Are there alternative causes (other than the drug) that could on their own have caused } \\
\text { the reaction? }\end{array}$ & -1 & +2 & 0 & +2 \\
\hline Did the reaction reappear when a placebo was given? & -1 & +1 & 0 & 0 \\
\hline Was the drug detected in blood (or other fluids) in concentrations known to be toxic? & +1 & 0 & 0 & 0 \\
\hline $\begin{array}{l}\text { Was the reaction more severe when the dose was increased or less severe when the } \\
\text { dose was decreased? }\end{array}$ & +1 & 0 & 0 & 0 \\
\hline $\begin{array}{l}\text { Did the patient have a similar reaction to the same or similar drugs in any previous } \\
\text { exposure? }\end{array}$ & +1 & 0 & 0 & +1 \\
\hline Was the adverse event confirmed by any objective evidence? & +1 & 0 & 0 & +1 \\
\hline Total Score & & & & 8 \\
\hline
\end{tabular}

*Score: Definite: $\geq 9$, Probable: 5-8, Possible: 1-8, Doubtful: o

Report: The suspected ADR found to be Probable on Naranjo scale assessment.

evaluation leads to suspect it as a drug induced psoriasiform and possibly metoprolol as a triggering agent as evident through literature reports. Metoprolol is a cardio-selective $\beta$ blocker used in the management of cardiac diseases. The potential role of medication to cause psoriasis is of clinical importance, especially in the patients who are treated for systemic comorbidities that are linked to psoriasis, such as hypertension and other cardiovascular diseases. There are numerous reports of $\beta$-blockers inducing and exacerbating psoriasis, $\beta$-2 receptors are found in keratinocytes, which can lead to decreased Cyclic adenosine monophosphate (cAMP) and consequent increases in intracellular calcium, causing increased cell proliferation with decreased differentiation. The latency period between drug exposure and psoriatic eruption can vary significantly, from several days to 48 weeks; if eruptions are indeed found, they are usually resistant to antipsoriatic medications until the $\beta$-blocker is discontinued. Probably every $\beta$-blocker is capable of provoking psoriasiform eruptions, aggravation or induction of the condition, Therefore, merely substituting one $\beta$-blocker for another may be unwise. For patients with psoriasis, $\beta$-blocker therapy is a particularly important issue. Recent evidence has shown an association between psoriasis and increased endothelial dysfunction and oxidative stress. Thus, the contraindication to $\beta$-blockers for these patients is only relative in the presence of absolutely indicated cardiac situations. ${ }^{7}$ The metoprolol in the present case was immediately replaced with telmisartan since the condition was getting worse with skin lesion all over the body and then, treated symptomatically using corticosteroid [hydrocortisone] and oral antibiotic [cefixime]. The patient condition improved after withdrawing metoprolol and lesions gradually healed on discharge.

A Naranjo causality assessment of the observed reaction reveals a "probable" causal relationship [Table 1] and rated under "moderate" severe adverse reaction category as per Hartwig's severity assessment.

\section{CONCLUSION}

The suspected ADR was found to have "probable" causal relationship with the suspected drug metoprolol as per Naranjo casualty assessment. Hartwig's severity assessment categorized the ADR under "moderately" severe adverse reaction category. Close monitoring of metoprolol usage among population for the occurrence of psoriatic adverse effect is warranted.

\section{ACKNOWLEDGEMENT}

The authors are thankful to the management of BLDE association for the required support.

\section{CONFLICT OF INTEREST}

The author declares no conflict of interest exists. 


\section{ABBREVATIONS}

TH1: T Helper Cell Type 1; TH2: T Helper Cell Type 2;

ESR: Erythrocyte sedimentation rate; $\mathbf{M C H}$ : Mean Corpuscular hemoglobin; RBS: Random blood sugar; GRBS: General Random Blood Sugar; cAMP: Cyclic adenosine monophosphate; ADR: Adverse drug reaction; IU: international unit.

\section{SUMMARY}

A patient with known hypertensive diagnosis on long standing use of beta blocker (metoprolol) to manage his hypertension, developed psoriasiform skin lesion which is attributed to and caused by metoprolol as drug induced reaction.

\section{REFERENCES}

1. Canpolat U, Yorgun H, Sunman H, Ulgen A, Aytemir K, Oto A. PP-152: BetaBlocker-Induced Psoriasis In A Patient With Heart Failure: A Case Report. International Journal of Cardiology. 2011;147(2):S137. DOI: https://doi. org/10.1016/S0167-5273(11)70379-5.

2. Puretić VM, Manc M, Ceović R, Lipozenčić J. Drug induced psoriasis. Acta Dermatovenerol Croat. 2011;19(1):39-42.

3. Adamu UG, Aisha A, Stephen AO, Joseph TC. Metoprolol-induced Psoriasiform Drug Eruption: A Case Report. International Journal of Medical and Pharmaceutical Case Reports. 2017;9(2):1-4. DOI: 10.9734/ IJMPCR/2017/33699

4. Zangeneh FZ, Shooshtary FS. Psoriasis-Types, Causes and Medication. 2013. http://dx.doi.org/10.5772/54728

5. Wu S, Han J, Li WQ, Qureshi AA. Hypertension, antihypertensive medication use and risk of psoriasis. JAMA Dermatol. 2014;150(9):957-63. doi: 10.1001/ jamadermatol.2013.9957

6. Balak DM, Hajdarbegovic E. Drug-induced psoriasis: clinical perspectives. Psoriasis (Auckland, NZ). 2017;7:87-94. doi:10.2147/PTT. S126727.

7. Hong J, Bernstein DBS. A review of drugs that induce or exacerbate psoriasis. Psoriasis. Forum. 2012;8(1):2-11 